Bull. Austral. Math. Soc.

VOL. 56 (1997) [453-458]

\title{
STIRRING OUR WAY TO SHARKOVSKY'S THEOREM
}

\author{
Seth Patinkin
}

\begin{abstract}
The periodic-point or cycle structure of a continuous map of a topological space has been a subject of great interest since A.N. Sharkovsky completely explained the hierarchy of periodic orders of a continuous map $f: R \rightarrow R$, where $R$ is the real line. In this paper the topological idea of "stirring" is invoked in an effort to obtain a transparent proof of a generalisation of Sharkovsky's Theorem to continuous functions $f: I \rightarrow I$, where $I$ is any interval. The stirring approach avoids all graph-theoretical and symbolic abstraction of the problem in favour of a more concrete intermediate-value-theorem-oriented analysis of cycles inside an interval.
\end{abstract}

\section{INTRODUCTION}

Let $X$ be a topological space, $f: X \rightarrow X$ a continuous map of $X$, and denote the $n^{\text {th }}$ iteration of $f$ by $f^{n}$. Let $k>1$. A point $p \in X$ is a point of period $n$ for $f$ in $X$ if $f^{m}(p) \neq p$, for $1 \leqslant m \leqslant n-1$ and $f^{n}(p)=p$. The points of the forward orbit of $p:\left\{p, f(p), \ldots, f^{n-1}(p)\right\}$ are said to form an $n$-cycle. In the early 1960's, Sharkovsky [7] elucidated the hierarchy of periodic orders for a continuous map of $R$, the real line. He discovered the following for $X=R$ :

Sharkovsky's Theorem. Assume $f: X \rightarrow X$ is a continuous function. If $p$ precedes $q$ in the following ordering of the natural numbers, then the existence of a p-cycle for $f$ in $X$ implies the existence of a $q$-cycle for $f$ in $X$ :

$$
3,5,7, \ldots, 2 \cdot 3,2 \cdot 5,2 \cdot 7, \ldots, 2^{2} \cdot 3,2^{2} \cdot 5,2^{2} \cdot 7, \ldots, 2^{2}, 2,1
$$

where $2^{j}(2 m+1)$ precedes $2^{k}(2 n+1)$ if $0 \leqslant j, k, m, n$ and exactly one of the following conditions is satisfied:
(i) $j=k$ and $1 \leqslant m<n$;
(ii) $j<k$ and $1 \leqslant m, n$;
(iii) $j>k$ and $m=n=0$;
(iv) $m>n=0$.

\section{Received 15th January, 1997.}

The author would like to thank C. Foias for his useful discussions and G. Cairns for suggesting the author write this paper.

Copyright Clearance Centre, Inc. Serial-fee code: 0004-9729/97 \$A2.00+0.00. 
It is well known that the converse of Sharkovsky's Theorem also holds. More precisely, for each natural number $n$, there exists a continuous function $f_{n}: J \rightarrow J$, where $J$ is a compact interval, such that the leftmost Sharkovsky period for $f_{n}$ in $J$ is $n[\mathbf{2}$, 7, 9]. Since his discovery, Sharkovsky's work has been advanced. Stefan [9] translated Sharkovsky's Russian paper into English in 1977. Straffin [10] offered a graph-theoretic proof of part of Sharkovsky's Theorem one year later. Block, Guckenheimer, Misiurewicz and Young [2] employed a similar Markov-graph representation of the periodic structure of $f$ to obtain a complete proof of the theorem in 1979. A paper of Ho and Morris [4] completed Straffin's work using his $k$-periodic digraph methodology as a means of interpreting the periodic structure of $f$ in 1981. Burkhart [3] completed Sraffin's work independently in 1982.

In addition to work on the original statement of Sharkovsky's result, there has been considerable work on generalising this notion of a periodic hierarchy to other spaces $X$. Block [1] succeeded in extending the Theorem to $X=S^{1}$, the circle, with the additional hypothesis that $f$ has a fixed point. Schirmer [8] succeeded in showing that Sharkovsky's Theorem remains true for $X=L$, a linear continuum, with the order topology. A linear continuum is a linear ordered set with more than one point such that

(i) $L$ has the least upper bound property,

(ii) $L$ is order dense, that is, if $x<y$, then there exists $z$ so that $x<z<y$. Munkres provides an introduction to this idea of linear continua in [6]. The generalisation of the original statement of Sharkovsky's Theorem to higher-dimensional Euclidean space is impeded by the need for the order relation of a linear continuum. However, Kloeden [5] discovered a generalisation to $X=C$, a compact subset of $R^{n}$, with the additional hypothesis that $i^{\text {th }}$ component of $f$ depends on the first $i$ independent variables.

\section{A STIRRING PROOF}

We shall show that Sharkovsky's Theorem holds for $X=I$, where $I$ is any interval, by use of the following topological notion of stirring. A point $a \in I$ such that

$$
\begin{gathered}
f^{2 k}(a)<\cdots<f^{4}(a)<f^{2}(a)<f^{2 k+1}(a) \leqslant a<f(a)<f^{3}(a)<\cdots<f^{2 k-1}(a) \\
{\left[\text { respectively } f^{2 k}(a)>\cdots>f^{4}(a)>f^{2}(a)>f^{2 k+1}(a) \geqslant a>f(a)\right.} \\
\left.>f^{3}(a)>\cdots>f^{2 k-1}(a)\right]
\end{gathered}
$$

will be called a $2 k+1$-stirring point. If $k=1$, we simply require $f^{2}(a)<a<$ $f(a)$ and $f^{3}(a) \leqslant a$ [respectively $f^{2}(a)>a>f(a)$ and $f^{3}(a) \geqslant a$ ]. If $a<f(a)$ in the above, then $a$ is referred to as an $u p 2 k+1$-stirring point. If $a>f(a)$ above, then $a$ is referred to as a down $2 k+1$-stirring point. If there is a $2 k+1$-stirring point for any $k$, we say there is stirring for $f$ in $I$. 
We first have the following:

PROPOSITION. If there is a $2 k+1$-stirring point for $f$ in $I$, then $f$ necessarily has points of all periods except for the odd numbers strictly between 1 and $2 k+1$.

ProOF: Fix an up $2 k+1$-stirring point $a \in I$. Assume that $f^{2 k+1}(a)<a$. Define $g(x)=f(x)-x$. Note that $g(a)>0>g(f(a))$ and there is thus a fixed point in $(a, f(a))$ by the intermediate value theorem. It remains to show

(i) the existence of points of all periods $N \geqslant 2 k$, and

(ii) the existence of points of periods $2 j$, for $1 \leqslant j \leqslant k-1$.

For convenience, define an index set $K=\{i \mid i \geqslant 2 k$ or $i=2 j$ for $1 \leqslant j \leqslant k-1\}$. We shall not distinguish between (i) and (ii); instead, we shall just consider the set $K$.

Let $f^{2 k}(a)=p_{0}$ and $f^{2 k-2}(a)=q_{0}$. Now, for $0 \leqslant j \leqslant k-2$, define:

$$
\begin{aligned}
& p_{2 j+1}=\sup \left\{x \in\left[f^{2(k-j)-3}(a), f^{2(k-j)-1}(a)\right) \mid f(x)=q_{2 j}\right\} \\
& q_{2 j+1}=\inf \left\{x \in\left(p_{2 j+1}, f^{2(k-j)-1}(a)\right] \mid f(x)=p_{2 j}\right\}, \\
& q_{2 j+2}=\inf \left\{x \in\left(f^{2(k-j-1)}(a), f^{2(k-j-2)}(a)\right] \mid f(x)=p_{2 j+1}\right\} \\
& p_{2 j+2}=\sup \left\{x \in\left[f^{2(k-j-1)}(a), q_{2 j+2}\right) \mid f(x)=q_{2 j+1}\right\} .
\end{aligned}
$$

Also let

$$
\begin{aligned}
p_{2 k-1} & =\sup \left\{x \in(a, f(a)) \mid f(x)=q_{2 k-2}\right\}, \\
q_{2 k-1} & =\inf \left\{x \in\left(p_{2 k-1}, f(a)\right] \mid f(x)=p_{2 k-2}\right\}, \\
q_{2 k} & =\inf \left\{x \in\left(a, p_{2 k-1}\right) \mid f(x)=p_{2 k-1}\right\}, \\
p_{2 k} & =\sup \left\{x \in\left[a, q_{2 k}\right) \mid f(x)=q_{2 k-1}\right\} .
\end{aligned}
$$

Finally, define for $j \geqslant k$ :

$$
\begin{aligned}
& p_{2 j+1}=\sup \left\{x \in\left(q_{2 j}, p_{2 j-1}\right) \mid f(x)=q_{2 j}\right\}, \\
& q_{2 j+1}=\inf \left\{x \in\left(p_{2 j+1}, p_{2 j-1}\right) \mid f(x)=p_{2 j}\right\}, \\
& q_{2 j+2}=\inf \left\{x \in\left(q_{2 j}, p_{2 j+1}\right) \mid f(x)=p_{2 j+1}\right\}, \\
& p_{2 j+2}=\sup \left\{x \in\left(q_{2 j}, q_{2 j+2}\right) \mid f(x)=q_{2 j+1}\right\} .
\end{aligned}
$$

Clearly, covering properties of the stirring structure allow for all of the points defined to exist. Now let $I_{i}=\left[p_{i}, q_{i}\right]$, for $0 \leqslant i$. Note that $f\left(p_{j}\right)=q_{j-1}$ and $f\left(q_{j}\right)=$ $p_{j-1}$ for $j \geqslant 1$. Since $f\left(I_{j}\right)=I_{j-1}$, for $1 \leqslant j$, it is clear that $f\left(\operatorname{int}\left(I_{j}\right)\right)=\operatorname{int}\left(I_{j-1}\right)$, where int $\left(I_{i}\right)$ denotes the interior of $I_{i}$. Define $g_{i}(x)=f^{i}(x)-x$. Note first that 
$f\left(I_{0}\right) \supset\left[a, f^{2 k-1}(a)\right]$ so that $f^{i}\left(I_{i-1}\right) \supset I_{i-1}$ for $i \in K$. Moreover, for these $i, g_{i}$ must have a zero in int $\left(I_{i-1}\right)$ by the intermediate value theorem, as $g_{i}\left(p_{i-1}\right)<0$ and $g_{i}\left(q_{i-1}\right)>0$ for $i$ odd and $g_{i}\left(p_{i-1}\right)>0$ and $g_{i}\left(q_{i-1}\right)<0$ for $i$ even. To conclude that $i$ is the period of this zero for $f$, we simply observe that, by construction, the $I_{i}$ 's have disjoint interiors. In the case that $f^{2 k+1}(a)=a$, it may happen that $g_{2 k+1}\left(p_{2 k}\right)=0$, in which case $p_{2 k}$ is a point of period $2 k+1$. In the case of a down-stirring point, the proof is symmetric.

Before proceeding, let us consider a cycle $P=\left\{p_{i}\right\}$ with $\# P=N<\infty$. Let $q=\max \left\{p_{i} \mid f\left(p_{i}\right)>p_{i}\right\}$ and $p=\min \left\{p_{i} \mid f\left(p_{i}\right)<p_{i}\right\}$.

LEMma 1. Suppose $P$, as just described, is a cycle for $f$. If $p<q$, then there is 3-stirring for $f$ in $I$.

PROOF: We will use the intermediate value theorem three times. Let $c_{2}$ be the rightmost fixed point in the interval $(p, q)$. Let $k=\min \left\{j \geqslant 1 \mid f^{j+1}(q)<c_{2}\right\}$. Find a preimage $c_{1}$ of $c_{2}$ in the interval $\left(q, f^{k}(q)\right)$. Last, find a preimage $c_{0}$ of $c_{1}$ in the interval $\left(c_{2}, c_{1}\right)$ and note that $c_{0}$ is an up 3 -stirring point for $f$ in $I$.

Lemma 2. Suppose that $P$ is an $N$-cycle for $f$ and $q<p$ as just described. If there exists an $i$ so that $p_{i}<f\left(p_{i}\right) \leqslant q$ (respectively, there exists a $j$ so that $\left.p \leqslant f\left(p_{j}\right)<p_{j}\right)$, then there is a $2 k+1$-stirring point for $f$ in $I$, for some $1 \leqslant k \leqslant\lfloor N / 2\rfloor$.

PROOF: Assume $p_{i}<f\left(p_{i}\right) \leqslant q$ for some $i$. Let $a_{0}=\min \left\{p_{i} \in P \mid p_{i}<f\left(b_{i}\right) \leqslant\right.$ $q\}$. Now define for $i \geqslant 0$ :

$$
\begin{aligned}
& a_{2 i+1}=\min \left\{p_{i} \in P \mid f\left(p_{i}\right) \leqslant a_{2 i}\right\} \\
& a_{2 i+2}=\max \left\{p_{i} \in P \mid f\left(p_{i}\right) \geqslant a_{2 i+1}\right\}
\end{aligned}
$$

Let $h=\min \left\{i \geqslant 1 \mid f\left(a_{0}\right) \leqslant a_{2 i}\right\}$. By construction,

$$
a_{0}<a_{2}<\cdots<f\left(a_{0}\right) \leqslant a_{2 h} \leqslant q<a_{2 h-1}<\cdots<a_{3}<a_{1} .
$$

Since $\# P=N$, it is clear that $1 \leqslant h \leqslant\lfloor N / 2\rfloor$. The intermediate value theorem allows us now to define for $i \geqslant 0$, setting $b_{0}=a_{0}$,

$$
\begin{aligned}
& b_{2 i+1}=\sup \left\{x \leqslant a_{2 i+1} \mid f(x)=b_{2 i}\right\} \\
& b_{2 i+2}=\inf \left\{x \geqslant a_{2 i+2} \mid f(x)=b_{2 i+1}\right\} .
\end{aligned}
$$

Note that $q \leqslant b_{2 i+1}$ and $p \leqslant b_{2 i+2}$ for $i \geqslant 0$. Let $k=\min \left\{i \geqslant 1 \mid f\left(a_{0}\right) \leqslant b_{2 i}\right\}$. Note that $b_{0}<b_{2}<\cdots<f\left(a_{0}\right) \leqslant b_{2 k}<q<b_{2 k-1}<\cdots<b_{3}<b_{1}$. Since $f\left(a_{0}\right) \leqslant a_{2 j} \leqslant b_{2 j}$, it is clear that $k \leqslant h$, so that $b_{2 k}$ is an up $2 k+1$-stirring point with $1 \leqslant k \leqslant\lfloor N / 2\rfloor$. If there is a $j$ such that $p \leqslant f\left(p_{j}\right)<p_{j}$, we obtain down stirring in a symmetric way. 
Corollary. Suppose $P$ is a $2 n+1$-cycle for $f$ in $I$. Then there is a $2 k+1$ stirring point for $f$ in $I$ for some $1 \leqslant k \leqslant n$.

Proof: Assume there is no $2 k+1$-stirring for $f$ in $I$, for $1 \leqslant k \leqslant\lfloor(2 n+1) / 2\rfloor=$ $n$. Then all points of $P$ must change sides of $[q, p]$ under $f$, by Lemma 2 . Since $q$ changes sides by definition, we must have that $f^{2 k}(q)$ lies to the left of $q$. But $f\left(f^{2 k}(q)\right)=q$, so that $f^{2 k}(q)$ does not change sides of $[q, p]$ under $f$, proving the corollary.

This stirring characterisation of odd cycles will enable us to prove Sharkovsky's hierarchy of periodic orders for $f$. Our task is two-fold. First, we must establish the body of Sharkovsky's ordering: $2^{j}(2 m+1)$ precedes $2^{k}(2 n+1)$ in lexicographic order for $0 \leqslant j, k$ and $i \leqslant m, n$. Second we must elucidate the tail of Sharkovsky's ordering: it remains to show that the powers of 2 (i) appear in decreasing numerical order and (ii) follow all the natural numbers with odd divisors.

\section{THE BODY}

We must show that the existence of a point of period $2^{j}(2 m+1)$ implies the existence of a point of period $2^{k}(2 n+1)$ if exactly one of the following conditions is satisfied:

(i) $j=k$ and $m<n$;

(ii) $j<k$, for $0 \leqslant j, k$ and $1 \leqslant m, n$.

To show (i), simply note that a point of period $2^{j}(2 m+1)$ for $f$ is a point of period $2 m+1$ for $\mathbf{f}^{2^{j}}$, which is also a continuous function. Thus the Corollary establishes (i).

To show (ii), again note that a point of period $2^{j}(2 m+1)$ for $f$ is a point of period $2 m+1$ for $\mathbf{f}^{2^{j}}$. By the Corollary, there is stirring for $\mathbf{f}^{2^{j}}$. Since, by the Proposition, stirring for $\mathbf{f}^{2^{j}}$ implies all even periods for $\mathbf{f}^{2^{j}}$, it suffices that $2^{k-j}$ is an even number, establishing (ii).

\section{THE TAIL}

First we will show that the powers of 2 appear in decreasing numerical order. It suffices to show that period 2 implies period 1. Suppose that $\{a, b\}$ is a 2-cycle for $f$ in $I$ so that $a<b$. As in the proof of the Proposition, note that $g(x)=f(x)-x$ has a zero in $(a, b)$. To show that period $2^{k+1}$ precedes period $2^{k}$ for $k \geqslant 0$, note that what is period $2^{k+1}$ for $f$ is period 2 for $\mathbf{f}^{2^{k}}$ and what is period $2^{k}$ for $f$ is period 1 for $\mathbf{f}^{2^{k}}$. Since continuity is preserved under composition, we are done.

It remains to show that the powers of two follow all the natural numbers with odd divisors. Suppose there is a $2^{k}(2 n+1)$-cycle for $f$ in $I$. Then there is a $(2 n+1)$-cycle 
for $\mathbf{f}^{2^{k}}$, implying there is a $2 m+1$-stirring point for $\mathbf{f}^{2^{k}}$ for some $1 \leqslant m \leqslant n$. Stirring for $\mathrm{f}^{2^{k}}$ implies all even periods for $\mathrm{f}^{2^{k}}$. In particular, $\mathrm{f}^{2^{k}}$ must have points of periods $2^{j}$, for $j>0$. Thus $f$ must have points of periods $2^{j+k}$, for $j>0$. This takes care of all powers of 2 except $2^{i}$, for $0 \leqslant i<k+1$. But we already proved that the existence of a point of period $2^{j}$ implies the existence of a point of period $2^{i}$, for $i<j$.

\section{REFERENCES}

[1] L. Block, 'Periods of periodic points of maps of the circle which have a fixed point', Proc. Amer. Math. Soc. 82 (1981), 481-486.

[2] L. Block, J. Guckenheimer, M. Misiurewicz and L.S. Young, 'Periodic points and topological entropy of one dimensional maps', in Global theory of dynamical systems, Proceedings (Northwestern, 1979), Lecture Notes in Mathematics 819 (Springer-Verlag, Berlin, Heidelberg, New York, 1980), pp. 18-34.

[3] U. Burkhart, 'Interval mapping graphs and periodic points of continuous functions', $J$. Combin. Theory Ser B 32 (1982), 57-68.

[4] C.W. Ho and C. Morris, 'A graph-theoretic proof of Sharkovsky's Theorem on the periodic points of continuous functions', Pacific J. Math. 96 (1981), 361-370.

[5] P.E. Kloeden, 'On Sharkovsky's cycle co-existence ordering', Bull. Austral. Math. Soc. 20 (1979), 171-177.

[6] J.R. Munkres, Topology, a first course (Prentice-Hall, Inc., Englewwod Cliffs, NJ, 1975).

[7] A.N. Sharkovsky, 'Coexistence of cycles of a continuous map of a line into itself', Ukrain. Mat. Zh. 16 (1964), 61-71.

[8] H. Schirmer, 'A topologist's view of Sharkovsky's theorem', Houston J. Math. 11 (1985 385-395).

[9] P. Stefan, 'A Theorem of Sarkovskii on the existence of periodic orbits of continuous endomorphisms of the real line', Comm. Math. Phys. 54 (1977), 237-248.

[10] P.D. Straffin, Jr., 'Periodic points of continuous functions', Math. Mag. 51 (1978), 99-105.

\footnotetext{
Department of Mathematics

Indiana University

Bloomington IN $\mathbf{4 7 4 0 6}$

United States of America

e-mail: spatinki@indiana.edu
} 